\title{
The Generation of Shared Cryptographic Keys through Half Duplex Channel Impulse Response Estimation at $60 \mathrm{GHz}$
}

\author{
Michael A. Forman, ${ }^{1}$ Senior Member IEEE Derek Young ${ }^{2}$
}

\begin{abstract}
Methods to generate private keys based on wireless channel characteristics have been proposed as an alternative to standard key-management schemes. In this paper, we discuss recent results in the implementation of a system for the generation of private keys for cryptographic communications using channel impulse-response estimation at $60 \mathrm{GHz}$. The testbed is composed of commercial millimeter-wave VubIQ transceivers, laboratory equipment, and software implemented in MATLAB. Novel cognitive enhancements are demonstrated, using channel estimation to dynamically change system parameters and estimate cryptographic key strength. We show for a complex channel that secret key generation can be accomplished on the order of $100 \mathrm{~kb} / \mathrm{s}$.
\end{abstract}

\section{Introduction}

Private-key cryptography employs a class of algorithms that use an identical or trivially related key for both encryption and decryption. Because this key must be private between, yet distributed among, communicating nodes, a secure key-distribution infrastructure is required. In scenarios where such an infrastructure is infeasible, several alternative methods for managing keying variables have been proposed, one of which utilizes the communications channel itself to generate a keying variable [1]. A channel is considered to be sufficient for such a purpose if the time required to deduce the channel characteristics are on the order of time required to do a brute-force search for the cryptovariable.

In a prior publication by the authors, a survey of demonstrated systems was performed and a generalized system was proposed [2]. The need for a cognitive system was introduced, to address the effects of jamming, the impact of constant sampling rates, the need for channel complexity and variation. This work builds upon that foundation by defining and demonstrating such a cognitive system.

\section{System Overview}

A block diagram of a cognitive node capable of creating shared private keys through channel measurements is shown in Figure 1. The system is composed of a key-generation block and a cognitivecontrol block which work together to improve and estimate the strength of generated keys.

The key-generation block converts received signals into cryptographic keys and consists of five sections. The channel-measurement section records and forwards data for analysis and key generation. The weight-generation section normalizes the data, converting it to vectors for demodulation. The keygeneration section uses a constellation with symbols to convert vectors into binary data. The keyvalidation section uses a hash to permit the exchange and validation of a generated key between nodes, using previously published methods [3]. Finally, the key-strength section stores a key with its estimated strength for use and comparison against future keys to identify degenerate (recurring) channel states.

For a channel to be suitable for use as a keying variable, it must be reciprocal, sufficiently complex, varying within bounds, and not in a degenerate state. While resistance of this key-generation method to eavesdropping is often discussed, the vulnerabilities introduced by the absence of the above channel requirements are often ignored.

In an attempt to address these vulnerabilities and to improve the performance of the system, a cognitive-control block is introduced, composed of three parallel paths. The measurement-control section analyzes the measured channel, to look for reciprocity and occupancy and to identify a subset of usable data for key generation. The constellationcontrol section selects the method of converting data to vectors and the type of constellation. The expiration-control section estimates the strength of the generated key based on channel characteristics.

\footnotetext{
1 Sandia National Laboratories, P.O. Box 969, MS9102, Livermore, California, 94551-0969, USA

Email: Michael.Forman@Sandia.GOV, Phone: +1-925-294-6188, FAX: +1-925-294-1539

2 Email: Derek.Young@Sandia.GOV, Phone: +1-925-294-3725, FAX: +1-925-294-1539

Sandia is a multiprogram laboratory operated by Sandia Corporation, a Lockheed Martin Company, for the United States Department of Energy's National Nuclear Security Administration under contract DE-AC04-94AL85000. SAND2009-7181C approved for unlimited public release.
} 


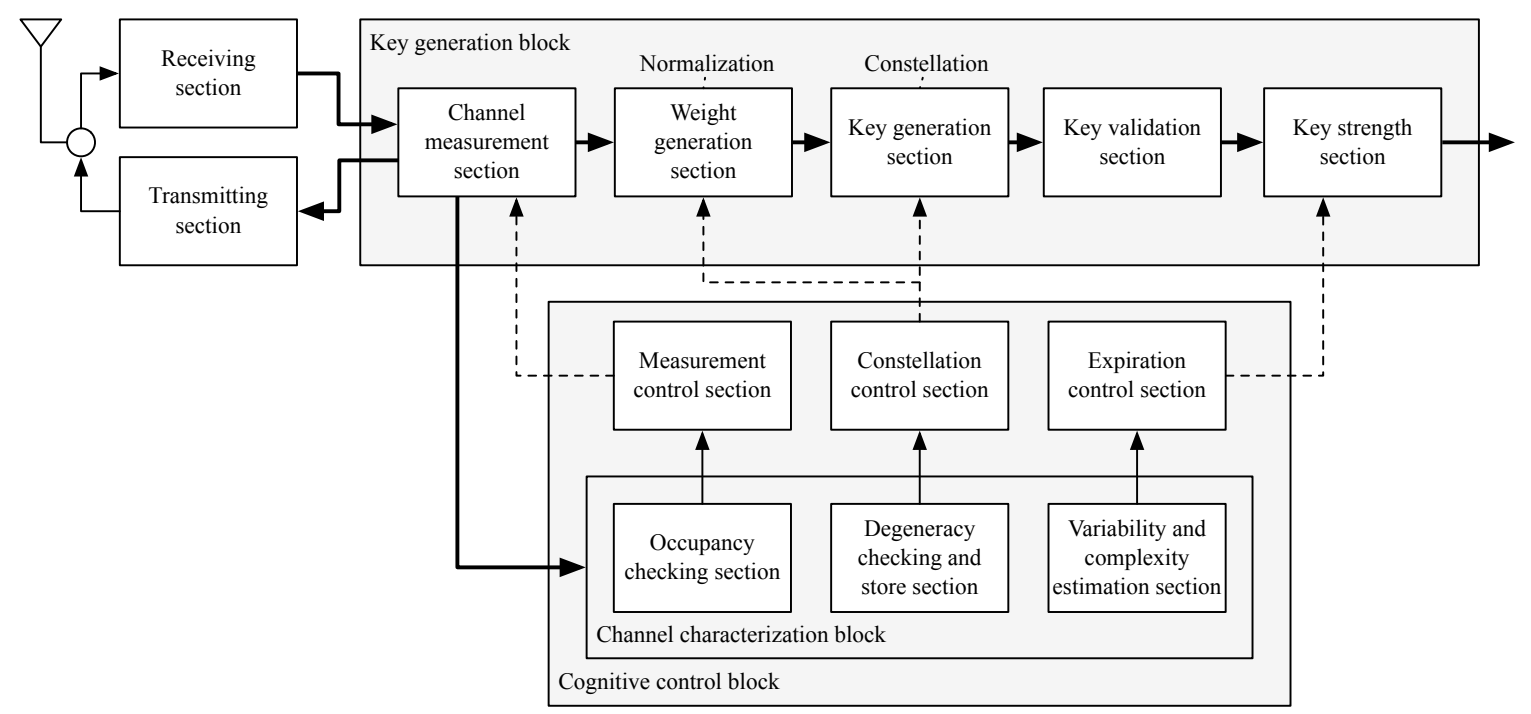

Figure 1: A block diagram of a cognitive node capable of creating shared private keys through channel impulse response estimation. The system is composed of a key-generation block and a cognitive-control block, which work together to improve and estimate the strength of generated keys.

\section{Half-Duplex Hardware Implementation}

A half-duplex testbed has been implemented to demonstrate elements of the proposed keygeneration system. The testbed consists of two transceivers, laboratory test equipment, and a single computer running MATLAB for control. The transceivers are the $60 \mathrm{GHz}$ VubIQ V60DSK01 with integrated digital control and antennas, selected for their $1.5 \mathrm{GHz}$ bandwidth, allowing the differentiation of path lengths that differ by as little as $60 \mathrm{~cm}$. This enables a detailed measurement of channel complexity, important in the evaluation of generated key strength.

Using previously published techniques [4], the system estimates the impulse response of a channel by repeating series of $1023 \mathrm{~b}$ pseudorandom numbers (PN), modulated on to the $60 \mathrm{GHz}$ carrier. The PNs are transmitted at $500 \mathrm{Mb} / \mathrm{s}$, repeating approximately every $2 \mathrm{~ms}$, and providing a 2 ns channel time resolution. The PN sequence length is tradeoff between signal gain and clock drift. Because the key-generation section makes use of phase information to generate data, the sequence is short enough to prevent the accumulation of significant phase errors from clock drift between the receiver and transmitter. Additionally, the system averages five consecutive channel measurements together, with phase normalized to the peak return, to reduce noise. Thus the system has a sampling period of $10 \mathrm{~ms}$, setting the upper bound on the variability that an environment can possess and still be characterized.

\section{Measurements}

The following measurements were performed with the half-duplex testbed while demonstrating latebreaking results at the 16th ACM Conference on Computer and Communications Security on 11 November 2009. A total of 57 measurements were taken in three sessions, each in distinct environments. The transmitter and receiver were separated by a distance of approximately $2 \mathrm{~m}$ and oriented in the same direction into a large room. Because of their configuration, each channel impulseresponse estimate includes an initial pulse representing the line-of-sight link between the transmitter and receiver - useful returns reflect from objects and return to the receiver. A single measurement from this session is presented initially to demonstrate the key-generation process, followed with an analysis of the entire data set and performance of the system in various environments.

\subsection{Single Measurement}

The power-delay profile of measurement number 41, made in a complex environment, is shown in Figure 2a. Phase information is omitted due to space constraints. This data originates in the channel-measurement section and is passed to the measurement-control section for the definition of a key-generation window.

The key-generation-window start time is $2.5 \mathrm{~ns}$ 
after the unchanging line-of-sight peak. The stop time is $53.3 \mathrm{~ns}$ after the start time, corresponding to a path length of $16 \mathrm{~m}$, an approximate limit imposed by transmitter power and receiver sensitivity. The lower power bound is set to be one standard deviation above the mean of the noise floor as measured over a $200 \mathrm{~ns}$ period preceding the initial peak. The upper power bound is the maximum power present within the window. The dynamic range of the shown key-generation window is $17.1 \mathrm{~dB}$.

The data in the key-generation window is passed to the weight-generation section which renormalizes the data (Figure 2b) and creates an array of vectors. The path of the vectors over the constellation is shown in Figure 2c. Each vector sample is converted to a $4 \mathrm{~b}$ value corresponding to one of the 16 symbols. In this measurement, 269 vectors generate $1076 \mathrm{~b}$ of data from the 16 -symbol constellation in $10 \mathrm{~ms}$. This is a data generation rate of $107.6 \mathrm{~kb} / \mathrm{s}$. The key validation-section is skipped in the half-duplex system.

The cognitive-control section estimates key strength using a weighted average of the keygeneration-window dynamic range, the powerdelay-profile complexity, and the key randomness estimated from a runs test. The key strength is stored as metadata with the generated key and used to set an expiration time for the key. Values for measurement number 41 are shown in the following subsection.

\subsection{Multiple Measurements}

The key-generation-window dynamic range, the key-generation-window complexity, and the estimated key randomness for all 57 measurements are shown in Figure 3. The measurements were taken in three environments, labeled A, B, and C. The single, previously presented measurement, number 41 , is marked with an inverted triangle.

Measurements in environment A were taken between 15:00 and 15:30 and represent a period of system setup, measuring close, static objects. Measurements in environment B were taken between 15:30 and 17:30 during system debugging in a large, empty room before the demonstration session. Measurements in environment $\mathrm{C}$ were taken between 18:00 and 19:00 in the same room filled with many moving conference attendees.

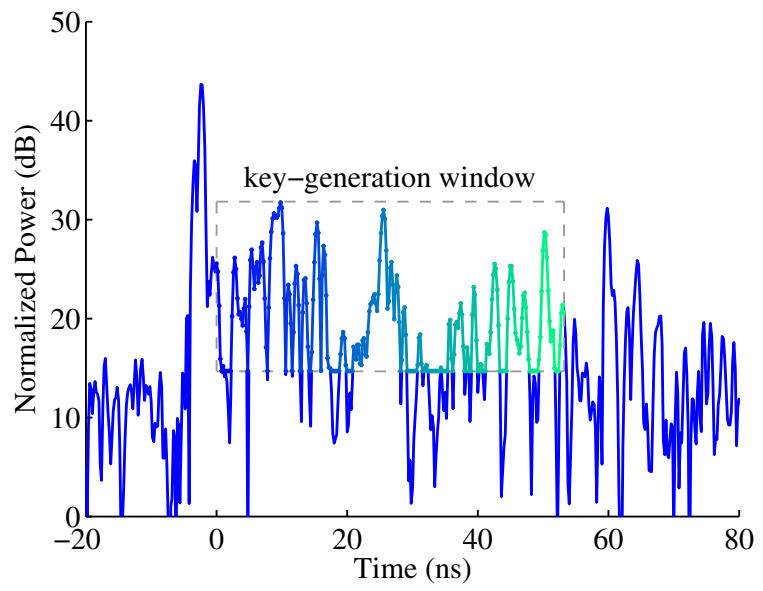

(a)

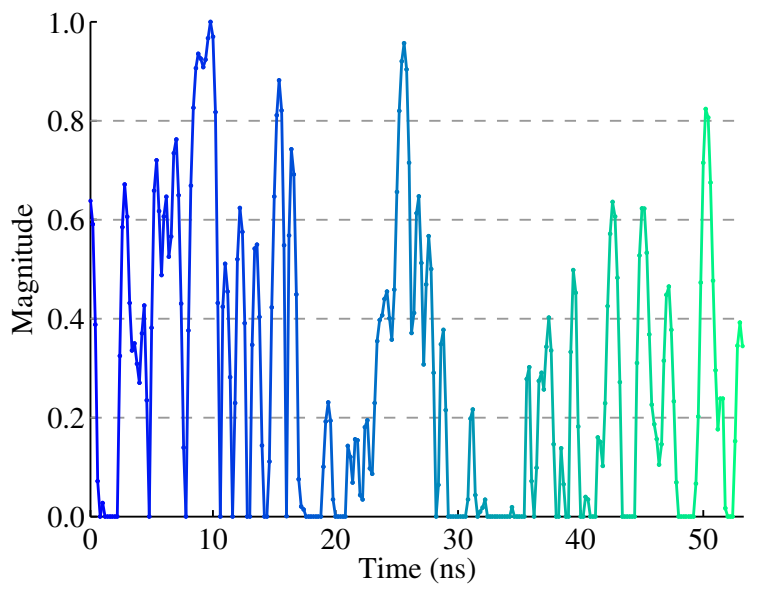

(b)

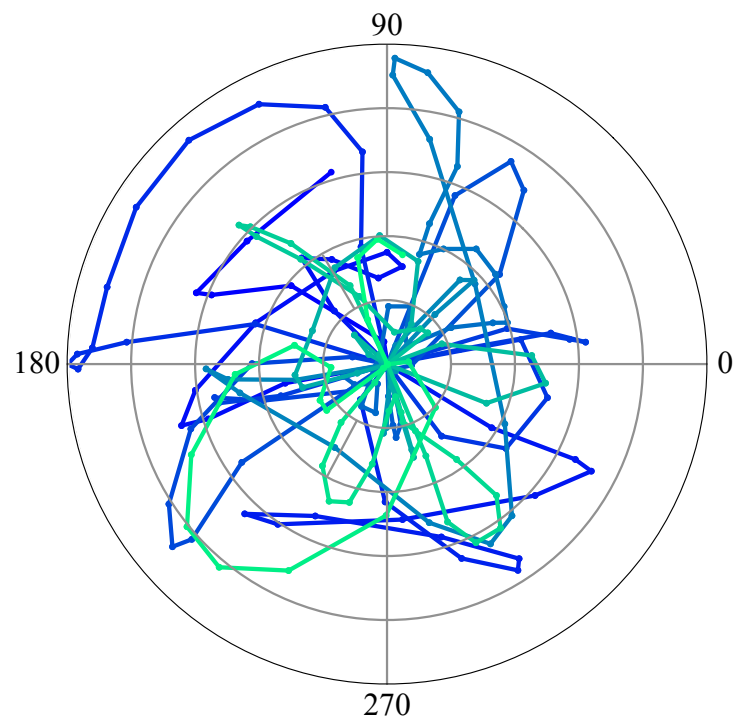

(c)

Figure 2: The power-delay profile of measurement number 41 (a), the normalized vector magnitude from the key-generation window (b), and the path over the 16-symbol constellation (c). 

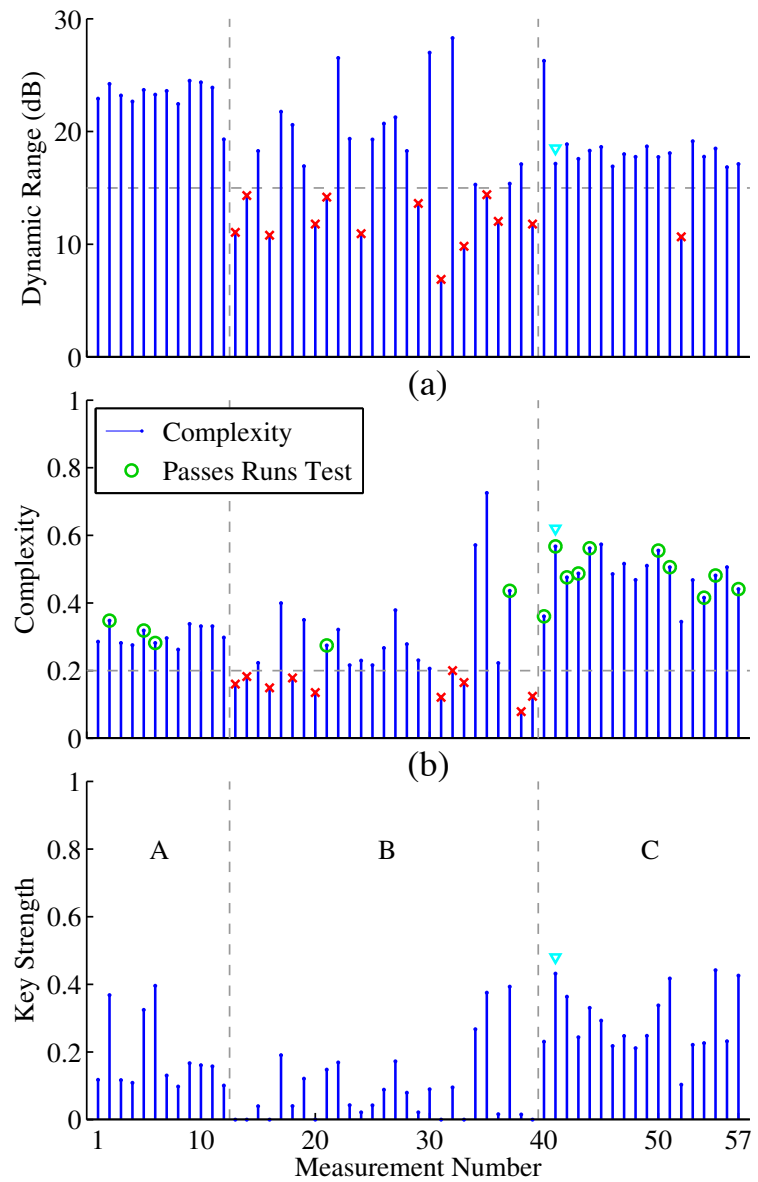

(c)

Figure 3: The key-generation-window dynamic range for all measurements (a), the key-generationsignal complexity and runs-test results (b), and the estimated key strength (c).

Studying the dynamic range, it can be seen that the close objects in environment A yielded a relatively high dynamic range due to the close proximity of objects, while the large, empty room of environment $\mathrm{B}$ did not. Outliers in the dynamic range in environment $B$ are due to reflections from group members moving randomly about during the testbed setup. Environment $\mathrm{C}$ with a full room of interested onlookers has a higher dynamic range than the same empty room that is environment B.

The system estimates complexity by calculating the ratio of areas above and below the signal in the key-generation window. This method identifies impulse responses with a large dynamic ranges that are nonetheless relatively simple, differentiating between one return and many returns. As one would expect, the full room of environment $\mathrm{A}$ is more complex (has more returns) than the empty room that is environment $\mathrm{B}$.

Key strength is estimated using a weighted average of the the key-generation-window dynamic range, the key-generation-signal complexity, and the calculated p-value from a runs test to measure randomness. Keys which passed the runs test are indicated with circles in the complexity plot in Figure 3b. Key strength estimates in the three environments agree with logically expected results, in that complex and varying environments provide stronger keys than simple and static environments.

\section{Conclusions}

Methods to generate private keys based on wireless channel characteristics have been demonstrated as an alternative to standard key-management schemes. We have demonstrated a half-duplex testbed for the generation of private keys for cryptographic communications using channel impulseresponse estimation at $60 \mathrm{GHz}$. Further we have defined and implemented a prototypical cognitive testbed which can respond to variations in the environment by adjusting sampling methods and assigning key strengths. Future work will include the implementation of a full-duplex system and further development of the algorithms used to estimate channel complexity and key strength.

\section{References}

[1] J. Hershey, A. Hassan, and R. Yarlagadda. Unconventional cryptographic keying variable management. Communications, Jan 1995.

[2] M. A. Forman and D. Young. A generalized scheme for the creation of shared secret keys through uncorrelated reciprocal channels in multiple domains. International Conference on Computer Communications and Networks, pages 1-8, May 2009.

[3] B. Azimi-Sadjadi, A. Kiayias, A. Mercado, and B. Yener. Robust key generation from signal envelopes in wireless networks. Proceedings of the 14th ACM conference on Computer and communications security, Jan 2007.

[4] D. Tholl, M. Fattouche, R. J. C Builtitude, P. Melancon, and H. Zaghloul. A comparison of two radio propagation channel impulse response determination techniques. IEEE Transactions on Antennas and Propagation, 41(4):515-517, 1993. 